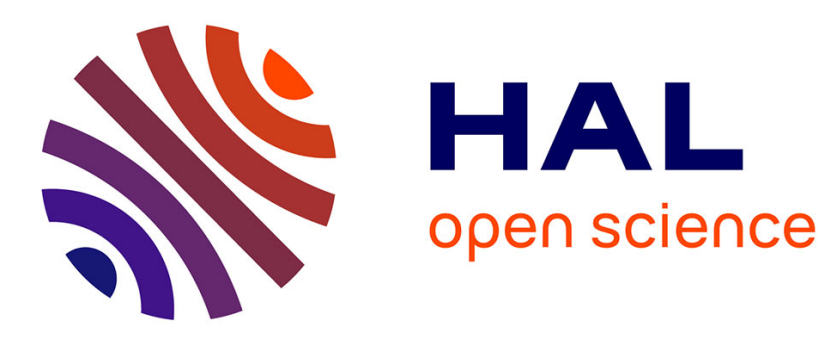

\title{
Surface-enhanced fluorescence imaging on linear arrays of plasmonic half-shells
}

\author{
Cosmin Farcau, Ana-Maria Craciun, Renaud A. L. Vallée
}

\section{To cite this version:}

Cosmin Farcau, Ana-Maria Craciun, Renaud A. L. Vallée. Surface-enhanced fluorescence imaging on linear arrays of plasmonic half-shells. Journal of Chemical Physics, 2020, 153 (16), pp.164701. 10.1063/5.0021906 . hal-02976227

\section{HAL Id: hal-02976227 \\ https://hal.science/hal-02976227}

Submitted on 23 Oct 2020

HAL is a multi-disciplinary open access archive for the deposit and dissemination of scientific research documents, whether they are published or not. The documents may come from teaching and research institutions in France or abroad, or from public or private research centers.
L'archive ouverte pluridisciplinaire HAL, est destinée au dépôt et à la diffusion de documents scientifiques de niveau recherche, publiés ou non, émanant des établissements d'enseignement et de recherche français ou étrangers, des laboratoires publics ou privés. 


\title{
Surface-Enhanced Fluorescence Imaging on Linear Arrays of Plasmonic Half-Shells
}

\author{
Cosmin Farcau ${ }^{1,2, *}$, Ana-Maria Craciun ${ }^{2}$, Renaud A. L. Vallée ${ }^{3}$ \\ ${ }^{1}$ National Institute for Research and Development of Isotopic and Molecular Technologies,
} 67-103 Donat Str., 400293, Cluj-Napoca, Romania

${ }^{2}$ Institute for Interdisciplinary Research in Bio-Nano-Sciences, Babes-Bolyai University, $42 \mathrm{~T}$. Laurian, 400271, Cluj-Napoca, Romania

${ }^{3}$ CNRS, Univ. Bordeaux, CNRS, Centre de Recherche Paul Pascal, UMR 5031, 33600 Pessac, France

*Email: cfarcau@itim-cj.ro

\begin{abstract}
Here, we perform a Surface-Enhanced Fluorescence (SEF) intensity and lifetime imaging study on linear arrays of silver half-shells (LASHS), a class of polarization-sensitive hybrid colloidal photonic-plasmonic crystal unexplored previously in SEF. By combining FLIM, scanning confocal fluorescence imaging, Rayleigh scattering imaging, optical microscopy, and FDTD simulations, we identify with high accuracy the spatial locations where SEF effects (intensity increase and lifetime decrease) take place. These locations are the junctions/crevices between adjacent half-shells in the LASHS, locations of high electromagnetic field enhancement, and strong emitter-plasmon interactions, as confirmed also by simulated field maps. Such detailed knowledge of the distributed SEF enhancements and lifetime modifications distribution, with respect to topography, should prove useful for improved future evaluations of SEF enhancement factors and a more rational design of efficiency-optimized SEF substrates. These linear arrays of metal-coated microspheres expand the family of hybrid colloidal photonic-plasmonic crystals, platforms with potential for
\end{abstract}


applications in optoelectronic devices, fluorescence-based (bio)chemical sensing or medical assays. In particular, due to the polarized optical response of these LASHS, specific applications as hidden tags for anti-counterfeiting or plasmon-enhanced photodetection can be foreseen.

\section{INTRODUCTION}

Fluorescence is widely used in sensing, optical devices, bioimaging, medical research and diagnosis. However, improving fluorescence sensitivity and efficiency, all the way to singlemolecule detection desirable in many applications, remains a big challenge. Surface- or metalenhanced fluorescence (SEF / MEF) enables the increase of molecular detection efficiency by altering the local optical electromagnetic (EM) environment of the emitter. Nanostructures with characteristic topographic features of the order of 10-100 nm are known to modify the spatial distribution of the EM field, an effect that is extremely pronounced in noble metal structures because of surface plasmon resonances (SPR), collective oscillations of conduction electrons ${ }^{1}$. The possibility to spatially concentrate light at the subwavelength scale and to locally enhance the intensity of EM fields at will, opened several new research directions and potential for developing novel applications.

In particular, the systematic application of EM field enhancement in SEF 2 is nowadays investigated in plasmonic metal nanostructures, with applications in sensing at the nanomolar level useful in bio-chemistry, medicine, pharmaceutics, homeland security, nanoscale light filtering and waveguiding useful in information technology, solar cells, LEDs and OLEDs for energy efficieny ${ }^{3-6}$. SEF was previously demonstrated on a diverse range of metal nanostructures: colloidal nanoparticles ${ }^{7}$, island films ${ }^{8}$, periodic structures made by conventional lithography ${ }^{9}$, periodic films obtained by colloidal self-assembly ${ }^{10}$. 
Integration in applications was possible and became desirable because of the observed positive effects of the light emitter-plasmon interactions, such as amplified emission intensity, improved photostability, improved energy transfer and/or changes in emission lifetime. In the same time, the newly observed phenomena propagated a change of paradigm in what was known on fluorescence: radiative transition rates can be modified, and are not intrinsic to the emitter (e.g. fluorophore molecule), the quantum efficiency can be raised, fluorescence can be directional ${ }^{11,12}$. Among the interesting results emerging from the interactions of emitters and surface plasmons, several very interesting effects should also be mentioned: fluorescence reemission by surface plasmons, in spectral regions in which the emission is weak, but plasmon resonances are strong ${ }^{13}$, polarized emission, controlled by plasmon-coupled resonances and not by the incident polarization ${ }^{14}$, super-emission, near-field spectra, single-photon beaming.

The emission enhancement observed in SEF results not only from the local field enhancement in terms of the excitation process, but also from an effect of the photon density of states on photon emission processes ${ }^{15}$, governed by the plasmon resonances of the structures. Although it is difficult to separate the effects of excitation and emission enhancements, the studies of fluorescence lifetime make it possible to dissociate these two contributions semi-quantitatively ${ }^{16}$. In this context, Fluorescence Lifetime Imaging Microscopy (FLIM) has proved to be a valuable tool to assess both changes of the environment in the direct vicinity of the emitters and the electromagnetic coupling occurring between emitters and plasmonic nanostructures ${ }^{17}$. In contrast to traditional imaging methods based on fluorescence intensity, FLIM provides improved accuracy due to the more sensitive emission lifetime parameter which depends strongly on the environment and binding events. In the same time, it is independent on the concentration and illumination intensity, except for specific cases where collective effects can take place ${ }^{18}$. FLIM technique is widely exploited in biology and medicine in applications related to environmental sensing, tissue characterization, 
detection of molecular interactions or materials science field in characterization of new materials and quality control assays. Still, despite of its remarkable abilities, there are only few reports on the use of FLIM in SEF studies on plasmonic nanostructures ${ }^{16,19-22}$. RitmanMeer et al. showed that the modifications to fluorescence lifetime and intensity by arrays of non-interacting Ag nanoparticles are spatially located at the particles' sites ${ }^{23}$. Ray et al. performed FLIM analyses on a Klarite SERS substrate consisting of inverted pyramidal pits to arrive to similar conclusions ${ }^{21}$. Li et al. on the other hand used FLIM to study SEF on single isolated gold nanoparticles, and observe the localization of intensity increase together with lifetime decrease $\mathrm{e}^{16}$.

In order to make relevant assessments and pertinent deductions from FLIM investigations, the nanostructures under study need to be rationally designed, have a wellknown, desirably ordered morphology. Moreover, in order to make correlations between the local SEF enhancement and the sample geometry/morphology, topographical features comparable to the wavelength are desired, in order to attain enough spatial resolution by confocal optical microscopy in FLIM. Beyond the fundamental purpose, in view of practical relevance, such nanostructures should also be cheap and robust and should be obtained through accessible approaches. Furthermore, we recall that in SEF, the main challenge still remains the control and design of surface structures which maximize the fluorescence enhancement of an emitter by adjusting the local EM field. Metal films over microsphere (MFoM) monolayer arrays represent a class of plasmonic materials that were demonstrated efficient for fluorescence enhancement ${ }^{24,25}$. The plasmon resonances they exhibit can be tuned from the UV to the IR range through the diameter (usually few hundreds $\mathrm{nm}$ ) of the dielectric colloidal spheres forming a lattice which is covered by thin (few tens of nm) metal films. Linear arrays of metal half-shells represent a particular version of MFoM in which the dielectric microspheres are arranged as parallel linear chains before metal coating. ${ }^{26}$ Such a 
nano-micro-plasmonic substrate with controlled morphology could prove very suitable for FLIM investigations of plasmonic effects and their correlations with local morphology.

In this work we combine FLIM, scanning confocal fluorescence imaging, and FDTD simulations to directly identify, with diffraction-limited resolution, the spatial locations which produce the highest SEF enhancements and fluorescence lifetime modifications in periodically nanostructured linear arrays of silver half-shells. The plasmonic substrates are fabricated by colloidal self-assembly on prepatterned substrates and metal coating by thermal evaporation. Their polarization sensitive plasmonic response is analyzed by optical reflectance measurements and FDTD simulations. FLIM investigations are performed for different sample configurations and incident polarizations, and correlated with optical microscopy, Rayleigh scattering imaging, and FDTD field profile maps, in order to provide correlations between local SEF effects (intensity and lifetime modifications) and local topography.

\section{EXPERIMENTAL}

Materials. The inner surface of a commercially available recordable Digital Versatile Disc (DVD-R) was used as substrate for the fabrication of the linear plasmonic nanostructures. Polystyrene spheres with $497 \mathrm{~nm}$ diameter in aqueous suspension $(2 \% \mathrm{~W} / \mathrm{v})$ were purchased from MicroParticles GmbH Germany. Nile Blue (NB) and polyvynil alcohol (PVA) was purchased from Merck Germany. The other reagents were of analytical grade and used without any further purification. Deionized water and/or high purity ethanol were used in all rinsing procedures.

Fabrication of Linear Arrays of Silver Half-Shells. First, the two sandwiched polycarbonate discs forming the DVD-R were mechanically separated, cleaned, and treated in 
UV-ozone to render the surface hydrophilic. Then, linear arrays of polystyrene colloidal microspheres were obtained by convective self-assembly (CSA) on the patterned inner surface of the DVD, from aqueous colloidal suspensions. The protocol employed to prepare the linear colloidal arrays and the home-built equipment used for CSA were described in detail elsewhere ${ }^{26}$. The resulting linear colloidal arrays were coated by silver films of $50 \mathrm{~nm}$ thickness, deposited by vacuum thermal evaporation. This produced arrays consisting of parallel linear arrangements of silver half-shells (HS) called here Linear Arrays of Silver Half-Shells (LASHS).

Characterization of the nanostructures. Morphological characterization of the nanostructures was done by standard optical microscopy, Scanning Electron Microscopy (SEM) on a Quanta 3D FEG- FEI Microscope and tapping mode Atomic Force Microscopy (AFM) on a WITec system. Polarized optical spectra were recorded with an Ocean Optics USB4000 UV-VIS spectrometer. For well-localized reflectivity measurements, the upright microscope of a WITec alpha300 R Raman microscope was used. A 10× / 0.25 NA objective was used to both illuminate and collect light, assuring that a negligible amount of scattered/diffracted light is being collected. The collected light was transmitted to the spectrometer via a $0.6 \mathrm{~mm}$ core diameter optical fiber, reducing the area of the sample being analyzed to a disk of about $60 \mu \mathrm{m}$ in diameter.

Preparation of samples for fluorescence studies. Different types of samples containing fluorophores were prepared in order to observe SEF effects in different experimental configurations. First, Nile blue (NB) was dispersed in a water-based polyvinyl alcohol (PVA) solution and spin-coated ( $\sim 50 \mathrm{~nm}$ thickness) on top of the LASHS. A second system was 
fabricated by spin-coating a NB-doped PVA film (NB-PVA) on a flat glass substrate, placing it on top of the metal nanostructures, and ensuring proximity by mechanical pressure.

Fluorescence measurements. Fluorescence lifetime imaging was performed on a MicroTime200 time-resolved confocal fluorescence microscope system (PicoQuant) using a picosecond diode laser head operating at $640 \mathrm{~nm} / 40 \mathrm{MHz}$ (laser power $0.24 \mathrm{~mW}$ ) as excitation source. The fluorescence signal, both excited and collected through a $100 \times / \mathrm{NA}=$ 0.9 objective, was spatially and spectrally filtered by a $50 \mathrm{~mm}$ pinhole and a HQ690/70 (Chroma) emission filter, respectively, before being focused on a PDM Single Photon Avalanche Diode (SPAD) from MPD. The detector signals were thereafter processed by a PicoHarp 300 Time-Correlated Single Photon Counting (TCSPC) data acquisition unit. The intensity and FLIM images were analyzed using SymPhoTime software. Scanning confocal fluorescence images were also recorded under $633 \mathrm{~nm}$ excitation line on a WiTec alpha300 R Raman microscope. The fluorescence signal was collected through a confocal pinhole of 100 $\mu \mathrm{m}$ diameter by using a $100 \times / 0.9$ NA objective. A typical scan was performed on a $10 \times 10$ $\mu \mathrm{m}^{2}$ area with a resolution of $100 \times 100$ pixels. Together with the relatively high scanning speed (i.e. short integration time, 0.1s/pixel) used, these conditions assured avoiding fluorophore photodegradation during the scan.

FDTD simulations. The numerical Finite Difference Time Domain (FDTD) simulations have been performed with Lumerical ${ }^{27}$. The structure has been designed in a way to mimic as closely as possible the real structure. Accordingly, a rectangular $5 \times 5$ finite array of polystyrene spheres $(500 \mathrm{~nm}$ diameter, $\mathrm{n}=1.59)$ has been arranged on a polycarbonate substrate $(\mathrm{n}=1.58)$. The $50 \mathrm{~nm}$ thick silver layer has been modelled by silver ellipsoids with radius along $\mathrm{x}, \mathrm{y}$, and $\mathrm{z}$ of 260,260 and $250 \mathrm{~nm}$, placed $50 \mathrm{~nm}$ higher than the polymer 
spheres, together with a $50 \mathrm{~nm}$ silver plate on the substrate, at the base of the arrangement of spheres, which has then been perforated by $500 \mathrm{~nm}$ diameter air disks $(\mathrm{n}=1)$. This structure has been excited by a plane wave launched from the top of the structure backwards in the $-\mathrm{z}$ direction. The excitation was polarized either along the chains (y direction) or perpendicular to it ( $\mathrm{x}$ direction). The transmittance and reflectance spectra were then acquired in $\mathrm{z}$ perpendicular planes respectively well below and above (in order to avoid near field effects) the structure. Electric fields profiles were also acquired in a z-cut plane located $100 \mathrm{~nm}$ above the equatorial plane of the arrangement of spheres, for both polarizations at the wavelength of experimentally observed NB emission $(675 \mathrm{~nm})$. For the recording of electric field profiles, a second set of simulations was alternatively performed, in which the incident light was focused owing to a numerical aperture $(\mathrm{NA}=0.25)$, to better approach the conditions used in the experimental fluorescence studies. In all simulations, an auto non-uniform mesh type with accuracy 3 has been used and the (Lumerical) Conformal Mesh Technology (CMT) was chosen, with the Yu-Mittra method $1^{28,29}$ applied to interfaces involving metals (the so-called conformal variant 2). To increase the accuracy and insure convergence, a sub mesh with step size of $10 \mathrm{~nm}$ was added all around the particles, and the grid size was even reduced to $5 \mathrm{~nm}$ in the gaps between the spheres. Perfectly Matched Layers were used on all sides of the simulation volume. The optical constants of silver are the ones provided by Rakić et al. ${ }^{30}$.

\section{RESULTS AND DISCUSSION}

\section{Sample morphology.}

Our linear plasmonic nanostructures consist of a silver film deposited on top of dielectric (polystyrene) microspheres arranged in a linear fashion along the parallel grooves of the DVD-derived substrate. On the microscopic, few-microns scale, and up to few millimetres, 
the DVD circular grooves pattern can be considered as made of linear parallel grooves. By convective self-assembly, in which the triple-contact line was aligned perpendicular to the DVD grooves and translated along them, the dielectric colloidal microspheres assembled inside the grooves. This was promoted by the deliberate matching of the microsphere size $(500 \mathrm{~nm})$ to the groove pattern period $(750 \mathrm{~nm})$. After the parallel chains of close-packed dielectric microspheres were metallized, the LASHS were obtained. The morphology of these samples is described by SEM images in Figure 1a,b and the cross-sectional scheme in Figure 1c.

\section{Polarization-dependent optical response.}

In order to highlight the strong polarization sensitivity of these linear plasmonic nanostructures, their optical properties have been analyzed by polarized reflectance microspectrophotometry. The reflectance spectra for light polarized parallel and perpendicular to the LASHS, at normal incidence, are presented in Figure 2a. As expected, based on the morphology of these metal nanostructures, possessing two orthogonal periodic characteristics and hence a high degree of asymmetry, their optical response is highly sensitive to the polarization of incident light. A high polarization contrast is notably observed within the region $650-900 \mathrm{~nm}$. The reflectance spectrum presents a broad maximum centered around 770 $\mathrm{nm}$ for perpendicular polarization, which is replaced by a minimum for parallel polarization. The reflectance ratio at, e.g., $770 \mathrm{~nm}$, reaches a factor of 10, in favor of the perpendicular component. The minimum reflectance at $750-780 \mathrm{~nm}$ for parallel polarization is reminiscent of the reflectivity minimum observed on the better-known metal-coated microsphere arrays with close-packed hexagonal symmetry ${ }^{31}$, and attributed to excitation of surface plasmons localized at the junctions between adjacent metal half-shells. Reflectance spectra were obtained also by FDTD simulations (Fig 2b), and these provide a good qualitative agreement 
with the experimental spectra. Also, quantitatively, the reflectance contrast between the two polarizations is in the same range as the experimental one. Next, the electric field intensity map within the LASHS, obtained for parallel polarization, at $675 \mathrm{~nm}$, in the range of the maximum emission of NB, is presented in Fig 2c. The highest field enhancement regions are located at the junctions between adjacent half-shells. For an excitation perpendicular to the linear HS chains (Fig 2d), only a minor enhancement is obtained near the edges of the silver half-shells. These metal half-shells have a sickle moon-like cross-section, therefore their thin edges can cause some additional localized enhancement.

\section{Fluorescence intensity and lifetime imaging.}

First, the sample consisting of NB-PVA films conformally coated on top of the LASHS (scheme in Fig 3a), was investigated by FLIM. The fluorescence intensity and fluorescence lifetime images are presented in Figure 3b-c. The images were obtained with the excitation laser polarization aligned parallel to the linear arrays of plasmonic half-shell, which are oriented vertically in these figures. First, the intensity image exhibits a clear pattern of highintensity spots, well-aligned vertically, and spaced by $500 \mathrm{~nm}$, the period of the LASHS. On the perpendicular direction (see inset of Fig 2b) the spacing corresponds to $750 \mathrm{~nm}$, the distance between the chains of half-shells. Based on the simulation results in Fig 2c, showing the locations of high electromagnetic field-enhancement in between adjacent Ag half-shells, we can tentatively attribute the locations of the higher fluorescence intensity to the junctions/crevices between adjacent metal half-shells. The lateral sizes of these bright spots in the intensity image are around $250-300 \mathrm{~nm}$, comparable to the size of an Airy disk for these experimental conditions. This diffraction-limited resolution was achieved due to the extreme, nanoscale localization of the plasmon-enhanced electromagnetic fields. Then, the fluorescence lifetime image in Fig 3c also presents a well-defined patterned of vertical stripe 
regions of alternating lower and higher lifetime values. The lower lifetimes coincide with the regions of higher fluorescence intensity, and are located along the linear chains of Ag halfshells. However, a much lower contrast is obtained in the lifetime image, indicating that fluorescence lifetime is reduced all over the sample surface; this is a consequence of the fact that in the regions between the parallel linear chains of half-shells some metallic stripe-like nanostructures exist on the substrate, which can also be responsible for plasmonic effects. Given also the quasi-3D nature of this type of sample, a certain averaging of measured lifetime can take place at each measurement point, due to fluorophores located at different nanometric distances and different sites within the laser focus. The overall average over the image in Fig 3c gives a lifetime of $\sim 1.0 \mathrm{~ns}$, significantly decreased, as compared to the lifetime of NB in a bare PVA film ( 2.9 ns). Some variability was observed when repeating this experiment, with measured lifetime obtained in the range 1.0-1.5 ns. We could attempt to attribute such small variations to the different focusing in different experiments, which can induce slightly different excitation and collection conditions, therefore addressing different populations of emitters.

Then, a sample consisting of a flat NB-PVA film sandwiched on top of the LASHS structure (scheme in Fig 3d) was investigated as reference providing the same emitters and same plasmonic structure, but in a different local configuration. First, a vertical stripe pattern (750 $\mathrm{nm}$ spacing) is visible in the fluorescence intensity image, however without resolved features along the half-shell chain. Secondly, the lifetime image on the other hand does not show any features, being rather uniform across the scanned area. Taking into account both observations, we would attribute the pattern observed in the intensity image to the different local reflectivity or scattering of the linear chains of half-shells and that of areas in between them. This difference creates a contrast contributing to either better excitation or collection efficiency, without a contribution of increased emission characteristic of SEF. In particular, this 
configuration with emitters located far from the junctions between adjacent half-shells does not allow for the observation of a decreased lifetime / increased emission intensity induced by an increased photonic local density of states. Moreover, the averaged lifetime over the image is around $2.8 \mathrm{~ns}$, very close to the one obtained on the bare NB-PVA film, in favor of our interpretation presented above. Note that NB lifetime depends on solvent (for solutions), and also on the polymer environment ${ }^{32}$, and this value is in the range of previous reports. To summarize this part, our results in Fig. 3 bring direct visual evidence of the importance of the precise location of the emitters causing well localized plasmon-enhanced emission and fluorescence lifetime modifications in SEF. For the conformally coated sample, with emitters filling the interparticle junctions, the observed lifetime modifications indicate that a plasmonic effect on the emission is acting to modify the dyes' radiative decay rates. Therefore, the enhancement of the emission intensity is a result of: i) excitation, through plasmon-enhanced electric fields; ii) emission, through the additional radiative decay rate channells brought by the metal nanostructures, which win over the ohmic losses, despite these being also increased due to the proximity of the metal surface.

Next, the effect of incident polarization on FLIM was explored. Figures $4 \mathrm{a}$ and $4 \mathrm{~b}$ present the fluorescence lifetime images obtained with the laser excitation aligned parallel, and respectively perpendicular to the LASHS. The well-defined pattern imposed by the plasmonic nanostructures is visible only for the parallel case. Although the average lifetimes exhibited in the two measurements/scans only slightly vary, the contrast between the two geometries clearly indicates the role of the excitation polarization in the overall fluorescence process. 
Finally, another experiment was dedicated to achieving a clear assignment of the locations of the SEF bright spots observed in Fig 3b. For this purpose, the sample was transferred to a confocal Raman microscope, on which, by $633 \mathrm{~nm}$ excitation (very close to the $640 \mathrm{~nm}$ excitation line used in FLIM experiments) and notch filter, fluorescence can be recorded and imaged. Furthermore, this equipment allows for multiple sample visualization and imaging modes, as described in the following. First, a sample area rich in defects, with incomplete chains of half-shells, was selected, to serve as an unambiguous spatial reference. Fig 5a presents an optical image of the analyzed sample area, in which such incomplete LASHS are visible. By adjusting the spectral center of the recorded spectrum, a very small portion of the Rayleigh scattered photons (laser) can be collected at the low frequency edge of the spectrum. Thus, an image can be constructed from the intensity of the Rayleigh scattering (/laser reflection) at each pixel of the scan. Such an image is presented in Fig 5b, for the sample area visualized in Fig. 5a. The extended bright areas are those without metal half-shells, where the flat metal film is obviously highly reflective. Along the chains of half-shells, the bright spots correspond to locations of half-shells, as confirmed by analyzing/ correlating this image together with Fig. 5a. Now, the fluorescence intensity image in Fig. 5c is obtained from the same data set, by plotting the spectral region of interest $(650-680 \mathrm{~nm})$, therefore the spatial correspondence between these three images is achieved with high precision. Note that, besides being diffraction-limited, the resolution of the images in Figure 5 is limited also by the 10 pixels/ $\mu \mathrm{m}$ of the raster scan. Few circles were superposed to the three images, marking the positions of spheres, as deduced from the optical microscopy image in Fig 5a. The positions of bright regions in the fluorescence intensity image fall exactly in between the positions of the bright spots from the Rayleigh scattering image. It is thus demonstrated that the locations of high SEF enhancement are the junctions between adjacent half-shells, as already guessed based on simulation results. Moreover, some spots of higher intensity can be 
observed in the fluorescence intensity image (marked by arrows), which again, are each time located in between half-shells. Additionally, it can be observed in Figure $5 \mathrm{c}$ that an enhancement is present for some half-shells on their sides free of neighbors, e.g. the single HS and the dimer marked at the center of the area. As already mentioned, the metal half-shells have a thin edge around the colloidal sphere circumference, and such thin edges can cause some additional electric field enhancement.

Linear arrays of metal half-shells represent a relatively new class of hybrid colloidal photonicplasmonic crystal, that can be seen as a further development of the more studied and better understood MFoM. We are not aware of any previous exploitation of LASHS in SEF studies, while several interesting papers provide many insights into the SEF behavior of MFoM. As an example, it was shown that by tuning the plasmon resonances of silver-coated microsphere arrays, controlled by sphere (half-shell) diameter, the SEF enhancement can be optimized ${ }^{25}$. The manipulation of strong coupling betweenthe Bragg-plasmon mode supported by an organo-metallic array and molecular excitons in the formof J-aggregates dispersed on the hybrid structure has been demonstrated both experimentally and theoretically ${ }^{33}$. On the same type of structure, the resonance modes have been elucidated in the far-field owing to narrowband fluorescence nano-reporters, numerical simulations, and electric near-field patterns obtained with a scattering type scanning near-field optical microscope (s-SNOM) ${ }^{34}$. Other authors demonstrated that also the less popular plasmonic metal copper can be efficiently used in copper-coated microsphere arrays for molecular fluorescence enhancement ${ }^{24}$. In a related but different approach, the silver half-shell array was formed on top of dye-doped dielectric microspheres ${ }^{35}$, leading to observation of spectral and directional reshaping of fluorescence as a result of coupling to photonic crystal Bloch modes and plasmons localized inside the silver caps. 
The results presented herein complete the above findings by a direct experimental demonstration of the subwavelength localization of SEF enhancement regions, and its dependence on topological surface features. We wish also to emphasize the important role played by ordered, polarization-sensitive SEF substrates in the identification of correlations between optical/plasmonic properties, topography, and SEF effects. From the practical point of view, such plasmonic platforms based on metal half-shells obtained by colloidal templating, without relying on complex and expensive nanofabrication equipment, could find use in optoelectronic devices (e.g. LEDs/ OLEDs), in fluorescence-based assays (e.g. those based on antibody - antigen interactions), homeland security or forensics (e.g. detection of illicit substances), or anti-counterfeiting (fluorescent hidden tags). The linear arrays of plasmonic half-shells presented here could prove particularly promising as hidden tags containing multiple codes selectively visible by one polarization or the other. As a note, our purpose was not to maximize the SEF enhancement factors of the plasmonic nanostructures, but merely to perform microscopic observations as the ones described, which are missing from the literature.

\section{Conclusions}

Linear arrays of silver half-shells (LASHS) were fabricated by convective self-assembly of colloidal dielectric microspheres on patterned dielectric substrates, followed by silver film coating. Both experimental and simulated optical reflectance of the LASHS present a strong polarization sensitivity to light polarized along or perpendicular to the chains of half-shells. By combining FLIM, scanning confocal fluorescence imaging, Rayleigh scattering imaging, optical microscopy, we identified with diffraction-limited resolution the spatial locations where SEF effects such as intensity increase and lifetime decrease take place. These locations 
are the junctions/crevices between adjacent half-shells in the LASHS, as confirmed also by FDTD electric field enhancement maps. Moreover, we highlighted the importance of the coupling between the emitters and the enhancement regions of the plasmonic film and of aligning the excitation polarization to the LASHS. To the best of our knowledge, such an imaging of SEF enhancement regions in periodic plasmonic nanostructures, is unique in the literature. Besides, the fluorescence maps offer a direct visual confirmation that, in SEF, only a small fraction of the totally exposed metallic surface provides the major part of the measured enhanced emission. Such detailed knowledge of the SEF enhancements distribution, with respect to topography, should prove useful for improved evaluations of SEF enhancement factors and a more rational design of efficiency-optimized SEF substrates in the near future. These linear arrays of metal-coated microspheres, like the larger family of hybrid colloidal photonic-plasmonic crystals, have potential applications in optoelectronic devices, fluorescence-based (bio)chemical sensing or medical assays. Due to their polarized optical response, specific applications as hidden tags for anti-counterfeiting or plasmon-enhanced photodetection can be particularly foreseen. Unlike the better-known metal-coated microspheres with hexagonal compact packing, these linear arrays exhibit controllablyaligned and precisely positioned hot spots (i.e. high enhancement sites at interparticle junctions) which can be individually addressed for fundamental SEF studies.

\footnotetext{
Acknowledgement. This work was supported by UEFISCDI, project PN-II-CT-RO-FR2014-2-0052, Brâncuși Programme for Integrated Actions.
}

Data availability. The data that support the findings of this study are available from the corresponding author upon reasonable request. 


\section{References}

${ }^{1}$ W.A. Murray and W.L. Barnes, Advanced Materials 19, 3771 (2007).

${ }^{2}$ E. Fort and S. Grésillon, J. Phys. D: Appl. Phys. 41, 013001 (2007).

${ }^{3}$ W. Deng, F. Xie, H.T.M.C.M. Baltar, and E.M. Goldys, Phys. Chem. Chem. Phys. 15, 15695 (2013).

${ }^{4}$ S.-M. Lee, Y. Cho, D.-Y. Kim, J.-S. Chae, and K.C. Choi, Advanced Optical Materials 3, $1240(2015)$.

${ }^{5}$ N.N. Lal, B.F. Soares, J.K. Sinha, F. Huang, S. Mahajan, P.N. Bartlett, N.C. Greenham, and J.J. Baumberg, Opt. Express, OE 19, 11256 (2011).

${ }^{6}$ H. Szmacinski, R. Badugu, F. Mahdavi, S. Blair, and J.R. Lakowicz, J Phys Chem C Nanomater Interfaces 116, 21563 (2012).

${ }^{7}$ M. Hernandez, G. Recio, R.J. Martin-Palma, J.V. Garcia-Ramos, C. Domingo, and P. Sevilla, Nanoscale Research Letters 7, 364 (2012).

${ }^{8}$ K. Aslan, Z. Leonenko, J.R. Lakowicz, and C.D. Geddes, J Fluoresc 15, 643 (2005).

${ }^{9}$ G. Vecchi, V. Giannini, and J. Gómez Rivas, Phys. Rev. Lett. 102, 146807 (2009).

${ }^{10}$ R.A.L. Vallée, M. Ferrié, H. Saadaoui, and S. Ravaine, Opt. Mater. Express, OME 2, 566 (2012).

${ }^{11}$ S.-H. Cao, W.-P. Cai, Q. Liu, and Y.-Q. Li, Annu Rev Anal Chem (Palo Alto Calif) 5, 317 (2012).

${ }^{12}$ Y. Zhang, K. Aslan, M.J.R. Previte, and C.D. Geddes, Appl. Phys. Lett. 90, 053107 (2007).

${ }^{13}$ H. Su, Y. Zhong, T. Ming, J. Wang, and K.S. Wong, J. Phys. Chem. C 116, 9259 (2012).

${ }^{14}$ T. Ming, L. Zhao, H. Chen, K.C. Woo, J. Wang, and H.-Q. Lin, Nano Lett. 11, 2296 (2011).

${ }^{15}$ X.-W. Chen, M. Agio, and V. Sandoghdar, Phys. Rev. Lett. 108, 233001 (2012). 
${ }^{16}$ J. Li, A.V. Krasavin, L. Webster, P. Segovia, A.V. Zayats, and D. Richards, Scientific Reports 6, 21349 (2016).

${ }^{17}$ J. Zhang, Y. Fu, and J.R. Lakowicz, J Phys Chem C Nanomater Interfaces 115, 7255 (2011).

${ }^{18}$ M. Gómez-Castaño, A. Redondo-Cubero, L. Buisson, J.L. Pau, A. Mihi, S. Ravaine, R.A.L. Vallée, A. Nitzan, and M. Sukharev, Nano Lett. 19, 5790 (2019).

${ }^{19}$ F. Xie, M.S. Baker, and E.M. Goldys, Chem. Mater. 20, 1788 (2008).

${ }^{20}$ E.A. Barnoy, D. Fixler, R. Popovtzer, T. Nayhoz, and K. Ray, Nano Res. 8, 3912 (2015).

${ }^{21}$ K. Ray and J.R. Lakowicz, J. Phys. Chem. C 117, 15790 (2013).

${ }^{22}$ L.-Y. Wei, K.-S. Huang, H.-H. Lin, Y.-P. Wu, K.-T. Tan, Y.Y. Lee, and I.-C. Chen, J. Phys. Chem. C 122, 28431 (2018).

${ }^{23}$ T. Ritman-Meer, N.I. Cade, and D. Richards, Appl. Phys. Lett. 91, 123122 (2007).

${ }^{24}$ K. Sugawa, T. Tamura, H. Tahara, D. Yamaguchi, T. Akiyama, J. Otsuki, Y. Kusaka, N. Fukuda, and H. Ushijima, ACS Nano 7, 9997 (2013).

${ }^{25}$ C. Farcău and S. Aştilean, Appl. Phys. Lett. 95, 193110 (2009).

${ }^{26}$ V. Saracut, M. Giloan, M. Gabor, S. Astilean, and C. Farcau, ACS Appl. Mater. Interfaces 5, 1362 (2013).

${ }^{27}$ (n.d.). https://www.lumerical.com/products/fdtd/

${ }^{28}$ Wenhua Yu and R. Mittra, IEEE Microwave and Wireless Components Letters 11, 25 (2001).

${ }^{29}$ A. Taflove, Computational Electromagnetics: The Finite-Difference Time-Domain Method (Artech House, Boston, 2005).

${ }^{30}$ A.D. Rakić, A.B. Djurišić, J.M. Elazar, and M.L. Majewski, Appl. Opt., AO 37, 5271 (1998).

${ }^{31}$ C. Farcau, M. Giloan, E. Vinteler, and S. Astilean, Appl. Phys. B 106, 849 (2012). 
${ }^{32}$ H.P.M. de Oliveira and M.H. Gehlen, Journal of the Brazilian Chemical Society 14, 738 (2003).

${ }^{33}$ P. Fauché, S. Ungureanu, B. Kolaric, and R.A.L. Vallée, J. Mater. Chem. C 2, 10362 (2014).

${ }^{34}$ S. Ungureanu, B. Kolaric, J. Chen, R. Hillenbrand, and R.A.L. Vallée, Nanophotonics 2 , 173 (2013).

${ }^{35}$ B. Ding, C. Hrelescu, N. Arnold, G. Isic, and T.A. Klar, Nano Lett. 13, 378 (2013). 


\section{FIGURE CAPTIONS:}

Figure 1. (a), (b) SEM images and (c) structural scheme of the linear arrays of Ag half-shells deposited on polystyrene $(P S)$ microspheres, themselves arranged on the polycarbonate $(P C)$ DVD substrate. (d) A transversal cut of the geometry used in simulations.

Figure 2. (a) Experimental and (b) simulated optical reflectance spectra for the parallel and perpendicular polarizations (see inset). Electric-field maps at $675 \mathrm{~nm}$, for the (c) parallel and (d) perpendicular excitation configurations.

Figure 3. (a) Scheme of NB-PVA film conformally coated on LASHS; (b) fluorescence intensity image, and (c) fluorescence lifetime image, for parallel polarization; (d) Scheme of NB-PVA flat film on glass sandwiched to the LASHS; (e) intensity image and (f) lifetime image.

Figure 4. Polarization effect on FLIM, with excitation laser polarized (a) parallel and (b) perpendicular to the linear nanostructures' axis.

Figure 5. Confocal scanning fluorescence microscopy observations: (a) optical image (photo) of a defects-rich area of the linear nanostructures; (b) Rayleigh-scattered laser light image; (c) scanning confocal fluorescence intensity image. The dotted circles indicate positions of metal half-shells, determined from panel (a), while arrows indicate the sites with the highest intensity. Area size is $6 \mu \mathrm{m} \times 6 \mu \mathrm{m}$. 


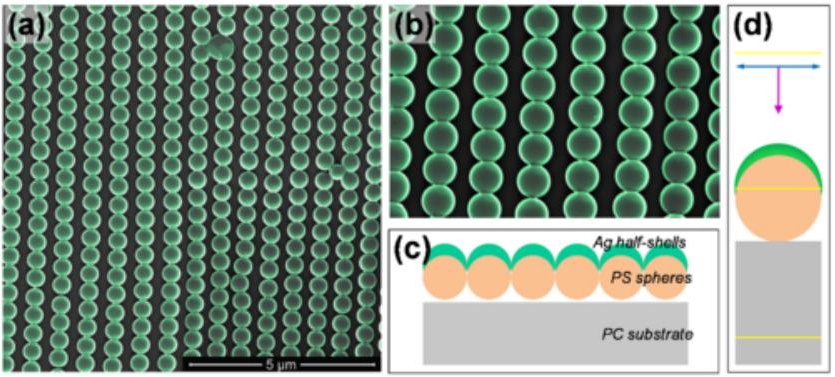




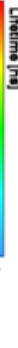


